\title{
Desarrollo y validación de una plantilla para la implementación de escenarios clínicos de simulación
}

\author{
Development and validation of a template for the \\ implementation of clinical simulation scenarios
}

\author{
Alejandro Martínez,* Elena Rojo, ${ }^{\ddagger}$ Liébana Piedra, ${ }^{\S}$ Ignacio del Moral,` José M Maestre**
}

\begin{abstract}
Palabras clave: Plantilla de escenario, simulación de pacientes, aprendizaje experiencial, objetivos de aprendizaje, debriefing.

Keywords: Scenario template, patient simulation, experiential learning, learning objectives, debriefing.
\end{abstract}

* Instructor de

simulación. Centro Simulación Avanzada, Ciudad Real y Hospital virtual Valdecilla,

Santander. Profesor

Escuela Enfermería

Fundación Jiménez Díaz,

España.

${ }^{\ddagger}$ Directora operaciones. Hospital virtual

Valdecilla, Santander.

$\S$ Instructora simulación.

Hospital virtual

Valdecilla, Santander.

" Director ejecutivo

Hospital virtual

Valdecilla, Santander.

** Director docente.

Hospital virtual

Valdecilla, Santander.

Recibido: 09/07/2019 Aceptado: 14/07/2019

\section{RESUMEN}

Introducción: Los objetivos docentes guían el diseño de un escenario simulado. El escenario proporciona a los participantes la experiencia concreta para el aprendizaje, y al instructor los datos sobre las acciones y resultados de los participantes que son la fuente para conducir el debriefing. Objetivo: Desarrollar una plantilla para guiar el diseño e implementación de escenarios simulados, y validar su capacidad para evocar los objetivos de entrenamiento planteados durante la simulación y el debriefing. Material y métodos: Se desarrolló una plantilla mediante un método Delphi modificado y se analizó su implementación tras el diseño de 30 escenarios de simulación creados por equipos multidisciplinares de diferentes países de Latinoamérica y España en un curso de formación de instructores. Resultados: La plantilla resultante consta de cinco fases secuenciales y cuatro elementos en cada una de ellas. Todos los escenarios implementados permitieron abordar durante el debriefing los objetivos de entrenamiento planteados. La fase 1 de enmarcación y la fase 4 de evolución fueron donde se detectaron mayor número de errores de implementación. Conclusión: La instauración de escenarios clínicos de simulación utilizando la plantilla descrita resulta eficaz para abordar los objetivos docentes definidos durante el caso simulado y durante el debriefing posterior.

\section{ABSTRACT}

Introduction: Learning objectives guide the design of a simulated scenario. The scenario provides participants with a concrete experience for learning, and instructors with data on participant actions and results that are the source to conduct the debriefing. Objective: Develop a template to guide the design and implementation of simulated scenarios and validate its ability to evoke the learning objectives during the simulation and debriefing. Material and methods: A template was developed using a modified Delphi technique and its implementation was assessed after the design of 30 simulation scenarios created by multidisciplinary teams from different countries in Latin America and Spain during a training course for simulation instructors. Results: The resulting template consists of five sequential phases and four elements in each of them. All the scenarios implemented allowed to address the specific learning objectives during the simulation and debriefing. A greater number of implementation errors took place during phase 1 (framing) and 4 (evolution). Conclusion: The implementation of simulation scenarios using the template was effective to address the defined learning objectives, both during the simulated case and during the subsequent debriefing.

\section{INTRODUCCIÓN}

$\mathrm{Z}^{\prime}$ proceso de aprendizaje empleando la Esimulación se comienza de modo ideal con una experiencia concreta en la que los participantes se involucran de manera plena y abierta. Luego, durante el debriefing reflexionan sobre los motivos de su actuación, lo observan desde muchas perspectivas, hacen comparaciones con las teorías existentes e integran nuevos conceptos y teorías. Por último, se motivan para emprender nuevas experiencias, probando activamente lo aprendido para tomar decisiones y resolver problemas, y de este modo reiniciar el ciclo. ${ }^{1}$

Así, los escenarios de simulación son un elemento clave para comenzar el ciclo de aprendizaje experiencial. Por un lado, permiten a los participantes generar experiencias clínicas similares a las que se producen en el contexto real, poniendo el foco en una actividad profesional o competencias específicas del cuidado del paciente, lo que ayuda a estructurar y guiar el proceso de aprendizaje. Es decir, el diseño de un escenario clínico está guiado por los objetivos de aprendizaje que se pretenden 
obtener. $^{2}$ Por otro lado, el escenario es también el momento cuando el instructor observa las acciones de los participantes y el resultado sobre el paciente en relación con los objetivos planteados, lo que proporcionará los datos para conducir el debriefing. ${ }^{3}$

Un escenario es la descripción de la interacción con el sistema de trabajo desde la perspectiva del profesional o equipo sanitario mientras atiende a uno o varios pacientes. En el sistema se incluye, además del paciente simulado (actor, maniquí, híbrido o paciente estandarizado), otros profesionales sanitarios, la familia o acompañantes, la tecnología, los espacios físicos, así como la organización del trabajo. ${ }^{4}$ Los escenarios generan experiencias clínicas para que los participantes apliquen las competencias establecidas por los objetivos de aprendizaje. $^{2}$

Para facilitar este proceso al educador, se ha publicado una amplia variedad de casos clínicos simulados tanto en revistas científicas como en repositorios de diverso origen; sin embargo, a menudo éstos no se adaptan a las necesidades particulares de aprendizaje, los recursos o al contexto específico donde tienen lugar los cuidados de salud, lo que obliga al educador a diseñar sus propios escenarios. El reto para los educadores es desarrollar escenarios de modo eficiente cuya implementación provoque reflexiones durante el debriefing alineadas con los objetivos docentes. ${ }^{5}$

Para dar respuesta a esta necesidad, diversos autores han descrito en la literatura formularios o plantillas que permiten describir de modo simple la interacción que tiene que ocurrir. Muchos de ellos se han validado para que el contenido requerido permita asegurar una comprensión básica de las tareas que debe soportar el sistema, así como el entendimiento de los usuarios y el contexto de trabajo. Los escenarios pueden derivarse de los datos recopilados durante las actividades habituales. Si no se tiene acceso a dichos datos, pueden escribirse escenarios basados en conocimientos previos o incluso en la mejor estimación; ${ }^{6}$ no obstante, no hemos encontrado estudios que evalúen si los escenarios implementados proporcionan la experiencia concreta a los participantes para la enseñanza y a la vez aportan al instructor los datos sobre las acciones y resultados de los participantes que son la fuente para conducir el debriefing. ${ }^{7} Y$ todo ello en relación con los objetivos de aprendizaje específicos que se pretenden. Ello permitiría disminuir el tiempo y carga de trabajo necesarios para llegar al diseño final, así como un debriefing más eficaz.

Así, el objetivo del presente estudio es desarrollar un plantilla o formulario para guiar el diseño de escenarios simulados, y validar su confiabilidad, es decir, su capacidad para entrenar a los participantes en los objetivos de aprendizaje en el tiempo y condiciones que fueron previstos.

\section{MATERIAL Y MÉTODOS}

\section{Desarrollo del contenido de la plantilla}

Para establecer un consenso de expertos sobre los elementos a incluir en la plantilla, así como sus descriptores (validez de contenido), se utilizó un método Delphi modificado. ${ }^{8}$ Se realizó un proceso iterativo de encuestas anónimas en el que se recurrió a siete instructores de simulación con al menos 10 años de experiencia educativa, realización de $>100$ escenarios de casos simulados en el ámbito interprofesional, procedentes de al menos cinco hospitales en Colombia y España. La participación fue voluntaria, sin ningún tipo de remuneración.

Inicialmente se revisaron las referencias de la literatura sobre diseño de escenarios y se hizo un resumen de las fases que con más frecuencia se utilizan para el guion del escenario. En una primera y segunda ronda de encuestas se interrogó acerca de fases consideradas más útiles para guiar la interacción de los participantes con el fin de permitir que se evoque el objetivo de aprendizaje, y se seleccionaron aquéllas en las que se alcanzó pleno consenso. Seguidamente se pidió valoración de la plantilla por cada instructor en el diseño de al menos cinco casos y se incorporaron las modificaciones en las que también hubo pleno consenso.

\section{Participantes}

Entre marzo de 2018 y marzo de 2019 se analizaron 30 escenarios de simulación diseñados e implementados, utilizando la plantilla durante una de las actividades del curso de 
formación de instructores de simulación clínica como herramienta docente del Institute for Medical Simulation. ${ }^{9}$ Se incluyó un total de 210 participantes (médicos, enfermeras, psicólogos y técnicos) originarios de Argentina, Chile, Colombia, Ecuador, España, México, Perú y Uruguay.

\section{Formación}

Se hizo una presentación teórica a los participantes previa a la utilización de la plantilla para comprender la importancia de evaluar las necesidades de aprendizaje, de definir los objetivos específicos de entrenamiento, seleccionar el tipo de simulador más adaptado a las tareas que se deben realizar con el paciente y determinar la mejor evidencia disponible para proporcionar un estándar de cuidado.

Además, se facilitó la comprensión de las fases de la evolución del caso, así como los elementos recogidos en la plantilla para tener en cuenta la interacción del equipo con el sistema durante el entrenamiento con el paciente. Previamente, los participantes experimentaron durante el curso tres escenarios diseñados siguiendo este mismo guion.

\section{Diseño del escenario}

El tiempo para diseñarlo fue de tres horas y se trabajó en grupos interprofesionales de siete personas. A cada grupo se le asignó de modo aleatorio un mandato para entrenar el trabajo en equipo durante la atención inicial al paciente grave, la toma de decisiones influida por aspectos ético-legales, así como la integración de las distintas perspectivas clínicas de todos los componentes de un equipo interprofesional. Cada grupo seleccionó además, un objetivo de aprendizaje clínico de su elección.

Los grupos consensuaron un caso clínico simulado para que un equipo interprofesional compuesto por siete personas manejara la situación y se entrenara específicamente en los objetivos planteados.

\section{Desarrollo del guion utilizando la plantilla}

Para guiar la interacción de los participantes durante la simulación se rellenaron, siguien- do las indicaciones de la plantilla, los datos correspondientes a la evolución del paciente, narrativa de los participantes embebidos en la simulación (también denominados confederados o actores) tanto sanitarios o pacientes como acompañantes, la disposición del contexto (espacio físico, tecnología y suministros necesarios para desarrollar la escena) y datos de la historia clínica necesaria.

\section{Ejecución del caso}

Los propios participantes ejecutaron el caso inmediatamente después de su diseño. Fueron apoyados por un equipo técnico de simulación y tuvo una duración que osciló entre 10 y 20 minutos. Cada escenario se realizó sólo en una ocasión.

\section{Evaluación de confiabilidad}

Inmediatamente después de cada escenario, los propios participantes que lo diseñaron realizaron un debriefing para analizar el rendimiento de sus compañeros.

La variable principal fue la capacidad del escenario implementado con la plantilla para evocar los objetivos de entrenamiento planteados. Los instructores, organizadores del curso, evaluaron durante el debriefing si se pudieron tratar o no los objetivos de aprendizaje clínico y/o de comportamiento, es decir, si los participantes pudieron reflexionar sobre los motivos de su actuación e integrar nuevos conceptos y teorías.

La variable secundaria fueron los errores ocurridos durante la implementación para alcanzar los objetivos de aprendizaje planteados. Para ello, posterior al debriefing se analizaron, junto con los participantes en el escenario, los aspectos que dificultaron su interacción con el paciente simulado y el resto del sistema para evocar los objetivos de aprendizaje en el tiempo y condiciones previstas.

\section{RESULTADOS}

La estructura de la plantilla para la implementación de escenarios clínicos de simulación resultante se recoge en la Tabla 1 . Consta de cinco fases secuenciales (enmarcación, con- 
textualización, presentación de los objetivos, evolución y finalización del caso) y cuatro elementos necesarios a considerar en cada una de ellas (paciente, confederados, historia clínica y contexto).

Los elementos incluyen 1) el tipo de paciente (actor, maniquí, paciente híbrido, paciente estandarizado o simulador virtual), los síntomas y signos vitales del paciente, así como el tipo de monitorización necesaria, accesos venosos y arteriales, suplementos de oxígeno u otros tratamientos aplicados (sondas, pulsioxímetro, etc.); 2) la narrativa y comportamientos que deben mostrar los confederados, es decir, los actores que no sean los pacientes que están programados en una simulación para proporcionar realismo, desafíos o información adicional para los participantes durante las distintas fases que dura el escenario (lo que puede incluir familia, acompañantes u otros intervinientes); 3) los datos correspondientes a la historia clínica que orienten a la evolución del paciente en cada fase (documentos escritos en papel o historia clínica electrónica); y 4) el contexto que corresponde a las características del lugar donde se lleva a cabo la asistencia al paciente, así como el equipamiento y los dispositivos tecnológicos necesarios.

En relación con las fases de implementación, la primera se denomina "enmarcación" y tiene como función proporcionar toda la información necesaria para orientar a los participantes sobre la situación: 1) identidad de la persona que avisa; 2 ) recuerdo del rol de los participantes; 3 ) condición clínica general del paciente y lugar donde se encuentra; $y$ 4) lo que se espera de los participantes. Esta etapa suele durar menos de un minuto. Es necesario tener presente dónde van a esperar los participantes (generalmente en la propia sala de debriefing) y cómo van a ser avisados (a menudo un sanitario que está al cuidado del paciente acude a llamarles). La enmarcación se puede hacer en la propia sala donde estén esperando los participantes o al ingresar éstos en la sala de simulación.

La segunda fase o de "contextualización" tiene como función permitir a los participantes situarse en el contexto de trabajo. Ello incluye dar tiempo a recabar los síntomas y signos vita-

Tabla 1: Plantilla para la implementación de escenarios clínicos de simulación.

Fase

(Duración recomendada)

Paciente

Confederados

Avisa a los participantes e informa sobre la situación

1. Enmarcación (1 min)

2. Contextualización (1-3 min)

3. Presentación de los objetivos

(1-10 min)

4. Evolución del caso

(5-10 min)

- Rendimiento

z estándar

- Rendimiento

$<$ estándar

5. Finalización

del caso

(1-5 min)

- Rendimiento

$\geq$ estándar

- Rendimiento

$<$ estándar
Síntomas y signos vitales iniciales

Evolución clínica para orientar hacia los objetivos de entrenamiento

Mejora o estabilización del paciente

Deterioro del paciente

Datos que señalen la mejoría o estabilidad clínica

La situación clínica no mejora o empeora
Narrativa y comportamientos de sanitarios, familia, acompañantes u otros intervinientes para orientar al contexto Narrativa y comportamientos de nueva información de sanitarios, familia, acompañantes u otros intervinientes que orienten a los objetivos

Narrativa y comportamientos consistentes con el rendimiento clínico de los participantes Narrativa y comportamientos consistentes con el rendimiento clínico de los participantes

El instructor finaliza el escenario y el paciente queda atendido por los confederados

Introducir un salvador del escenario previo a que el instructor finalice el escenario

Historia clínica

Contexto

Documentos necesarios en esta fase

Documentos necesarios en esta fase que faciliten la contextualización

Documentos necesarios en esta fase que orienten a los objetivos

Documentos necesarios en esta fase que orienten a la evolución clínica del paciente

Documentos necesarios en esta fase que orienten a la evolución clínica del paciente

Documentos necesarios en esta fase que orienten a la mejoría 0 estabilidad clínica

Documentos necesarios en esta fase que orienten a saber por qué el paciente no mejora
Lugar de espera de los participantes

Lugar de asistencia al paciente y características

Nuevos dispositivos que puedan necesitarse

Si se solicita y resulta necesario para el escenario, aportar nuevos dispositivos o tecnología Si se solicita y resulta necesario para el escenario, aportar nuevos dispositivos o tecnología Preparar contextos alternativos si la situación requiere trasladar al paciente

Si se necesita el salvador del escenario, aportar nuevos dispositivos o tecnología 
les del paciente, conversar con los confederados presentes (sanitarios, familia, acompañantes $u$ otros intervinientes) para orientarse en el contexto de trabajo y consultar los documentos disponibles en papel o formato electrónico con el objetivo de que tengan toda la información disponible del contexto donde se desarrolla el cuidado del paciente. La duración recomendada oscila entre uno y tres minutos, y depende de la situación clínica del paciente.

La tercera etapa o "presentación de los objetivos" tiene como función proporcionar los datos de la evolución clínica para orientar hacia los objetivos de entrenamiento. Esto incluye los síntomas y signos del paciente, la información que puedan proporcionar los confederados, así como los documentos necesarios de la historia clínica. La duración recomendada oscila entre uno y 10 minutos, y depende de la situación clínica del paciente. En una situación de crisis (por ejemplo, una parada cardiorrespiratoria) la segunda y tercera fases pueden estar solapadas.

La cuarta fase es de "evolución del caso" y será consistente con el rendimiento clínico de los participantes en relación con los objetivos planteados. Si el rendimiento observado es igual o superior al estándar de cuidado esperado, la evolución del paciente, la información proporcionada por los confederados y así como sus comportamientos orientarán a la estabilización o mejoría del paciente. En caso de que el rendimiento esté por debajo del estándar orientará al deterioro clínico. En cualquiera de estas dos situaciones el instructor obtiene datos observables que podrán ser utilizados durante el debriefing para descubrir los motivos que dieron lugar a esas acciones y resultados observados. Por otra parte, a menudo se solicita ayuda externa y pruebas complementarias en esta fase, y es necesario aclarar los recursos con los que cuentan para el cuidado del paciente.

La quinta y última etapa es la "finalización del caso", e igualmente depende del rendimiento clínico. Si éste coincide con el esperado y el paciente está estable, normalmente el instructor responsable finaliza el escenario y deja a los confederados a cargo del paciente. En caso de que el rendimiento se encuentre por debajo del estándar y la situación clínica del paciente se deteriore, una alternativa válida es introducir un salvador del escenario (por ejem- plo, un especialista avisado por el confederado que ayuda a estabilizar la situación) y finalizar el caso después. ${ }^{10}$

Todos los escenarios implementados con la plantilla permitieron a los instructores durante el debriefing conversar sobre los objetivos de entrenamiento planteados a los participantes (tanto los de comportamiento como clínicos). Los objetivos de comportamiento específicos fueron distintos de acuerdo a cada grupo (por ejemplo, claridad de roles o comunicación para trabajo en equipo; cuándo un paciente conserva el principio de autonomía en situación aguda; o cómo opiniones contrastadas de los profesionales pueden ayudar a orientar el diagnóstico de un paciente). Igualmente, cada grupo eligió distintos objetivos clínicos (por ejemplo, tratamiento de arritmias supraventriculares, indicación de transfusión en estados de shock o momento para realizar una cesárea en paciente con parada cardiaca).

Los grupos de trabajo señalaron, tras la evaluación de los escenarios, que todos ellos eran confiables para volver a ser utilizados y ninguno fue desechado para el futuro. Se detectaron errores de implementación y se sugirieron cambios en el diseño que oscilaron en un rango entre dos y siete para cada escenario. Los principales cambios sugeridos fueron: 1) en la fase uno, el modo en que se introdujo el contexto de trabajo (en cuatro escenarios que se desarrollaron simulando un contexto fuera del área clínica habitual); 2) en la fase dos, incongruencia entre los datos reflejados en los documentos, los narrados por los pacientes y los confederados (en cinco escenarios, nombre, edad y antecedentes); 3) en la fase tres, presentación de los objetivos antes de lo previsto (en un escenario, un familiar reveló la insulina como causa de la parada antes de lo esperado) y la falta de preparación de los documentos necesarios (en seis escenarios faltó la analítica y electrocardiograma); 4) en la penúltima fase, la interacción con los confederados (en siete escenarios no se siguió una fidelidad conceptual y emocional congruente con la actuación de los participantes); y 5) en la última fase, principalmente, se generaron dudas sobre el momento de finalizar el escenario (alargándose de manera innecesaria el caso en cinco ocasiones). 


\section{DISCUSIÓN}

El diseño e implementación de escenarios clínicos de simulación utilizando la plantilla descrita en el presente trabajo, resulta eficaz para abordar los objetivos docentes planteados por los educadores durante el caso simulado y durante el debriefing posterior. La plantilla permite guiar la interacción de los participantes con el sistema de trabajo mientras atienden a uno o varios pacientes, de modo que permite dirigir el proceso de aprendizaje mediante experiencias guiadas por los objetivos de aprendizaje que se pretenden obtener.

Hay numerosas sugerencias en la literatura para desarrollar escenarios clínicos de simulación; sin embargo, no es el objetivo de este artículo realizar una revisión exhaustiva, pero sí destacar las características de las plantillas disponibles más comúnmente utilizadas. Por lo regular se incluyen seis características durante el diseño como la información del participante (tipo y selección de los alumnos); los métodos docentes (identificación de objetivos y métodos para evaluarlos); estrategia de diseño (flujo de los eventos, transiciones y tipos de rendimiento esperado); material de apoyo (no sólo para el escenario, sino referencias bibliográficas y herramientas validadas de evaluación); generalización (para el tipo de disciplina y de simulador) y accesibilidad (de libre acceso online publicada en la literatura o restringida a la propia institución). ${ }^{6}$ El contenido de la plantilla o formulario presentado es similar al descrito en la literatura. Así, el flujo del escenario comúnmente se secuencia en una fase inicial, otra de "gatillo", una intermedia y una final. Los elementos para cada una de ellas por lo general incluyen los parámetros para evaluar la situación del paciente, los factores ambientales y los comportamientos esperados de los participantes. ${ }^{11}$ La mayoría de las propuestas están fundamentadas en la revisión de la literatura y se realiza a través de la creación de grupos de trabajo que acuerdan los componentes esenciales a incluir en la plantilla, de modo similar al método Delphi utilizado para el presente trabajo. ${ }^{12}$

El proceso de validación varía dependiendo de los grupos, siendo el método más frecuentemente utilizado la revisión y retroalimentación del escenario por un equipo de expertos que comprueban las referencias que apoyan los resultados esperados. A menudo se emplea una lista de verificación para comprobar que están incluidos todos los elementos necesarios para guiar la interacción de los participantes con el paciente (o pacientes) y el sistema de trabajo. En ocasiones se pilotan y prueban los escenarios para entender más en profundidad las interacciones antes de su utilización final con participantes, y así garantizar la consistencia de la experiencia clínica que se evoca. ${ }^{13}$ Otros métodos para validarlos incluyen tres fases consistentes en el desarrollo del escenario (revisando historias reales de pacientes, completando la plantilla y verificando el contenido por expertos), el pilotaje (probando los escenarios y refinándolos) y la implementación (haciendo encuestas a los participantes sobre fidelidad y el realismo vivido). ${ }^{14}$

El análisis realizado ha permitido identificar los momentos clave en el desarrollo del escenario que garantizan una orientación efectiva de los participantes al objetivo docente. De las tres fases iniciales, el punto más relevante fue la enmarcación al escenario, ya que una falta de orientación precisa al comienzo dificulta el desempeño posterior de los participantes. De las dos fases finales el punto más relevante correspondió a la fase cuatro, donde los confederados que no respondieron con fidelidad conceptual y emocional acorde al rendimiento de los participantes, prolongaron la evolución del caso o confundieron a los participantes. Los instructores que están dirigiendo el escenario desde la sala de edición pueden estar en contacto directo por audio con los confederados y ayudarlos a prevenir esta disociación entre el rendimiento de los participantes y la actuación de los confederados, manteniendo una respuesta acorde a la situación.

La principal diferencia de la plantilla aquí presentada con las otras descritas en la literatura, radica en el método de validación que, además de revisar el escenario por expertos y participantes, incluye la verificación de si realmente se puede realizar el debriefing sobre los objetivos de aprendizaje planificados. Los resultados muestran que permiten enfocar la experiencia de aprendizaje independientemente de la disciplina de los participantes. Además, los resultados son reproducibles cuando la plantilla se ha utilizado por instructores de simulación de distintos países de Latinoamérica y España.

Las implicaciones de la práctica son que para garantizar que la experiencia de aprendi- 
zaje se enfoque al objetivo específico, las tres fases iniciales de interacción de los alumnos con el paciente y el sistema de trabajo son independientes del rendimiento de los participantes. La adaptación de los componentes del escenario al rendimiento particular de cada grupo de alumnos se restringe a las fases de evolución y finalización del caso. Resulta importante anticipar las posibles alternativas del rendimiento del equipo en estas dos últimas fases para responder en tiempo y fidelidad adecuados según la respuesta de los participantes.

Otra de las ventajas encontradas tras la utilización de la plantilla fue la reducción del tiempo global para diseñar un escenario de simulación, ya que se invirtieron tres horas para realizarlo y los cambios necesarios tras un primer pilotaje se limitaron a un rango entre dos y siete cambios.

Una de las limitaciones de esta plantilla es que el guion es lineal o secuencial, y aunque las fases cuatro y cinco permiten anticipar los parámetros, si el rendimiento está por encima y por debajo del estándar de cuidado, puede no prever todas las posibles actuaciones de los participantes. Otra limitación, es la ausencia de un grupo control que utilice otra plantilla para comparar los resultados observados en los debriefings.

A la vez, ello es una oportunidad para diseñar estudios futuros que comparen la eficacia de distintos modelos de plantilla para conseguir los objetivos de aprendizaje deseados durante el debriefing, el número de pruebas necesarias, el número de errores detectados y el tiempo requerido para completar el diseño.

\section{CONCLUSIÓN}

En conclusión, esta plantilla facilita el diseño e implementación eficiente de escenarios de simulación para alinear la experiencia de aprendizaje con los objetivos de entrenamiento.

\section{REFERENCIAS}

1. Armstrong E, Parsa-Parsi R. How can physicians' learning styles drive educational planning? Acad Med. 2005; 80 (7): 680-684.

2. Maestre JM, Sancho R, Rabago JL, Martínez A, Rojo E, Del Moral I. Diseño y desarrollo de escenarios de simulación clínica: análisis de cursos para el entrenamiento de anestesiólogos. Educ Med. 2013; 16 (1): 49-57.
3. Rudolph JW, Simon R, Raemer DB, Eppich WJ. Debriefing as formative assessment: closing performance gaps in medical education. Acad Emerg Med. 2008; 15 (11): 1010-1016.

4. Maestre JM, Pedraja J, Herrero L, Cano M, Rojo E, Suárez C, et al. Simulación clínica para la mejora de la calidad en la atención a la hemorragia posparto. J Healthc Qual Res. 2018; 33 (2): 88-95.

5. Jarzemsky P, McCarthy J, Ellis N. Incorporating quality and safety education for nurses competencies in simulation scenario design. Nurs Educ. 2010; 35 (2): 90-92.

6. Benishek LE, Lazzara EH, Gaught WL, Arcaro LL, Okuda Y, Salas E. The Template of Events for Applied and Critical Healthcare Simulation (TEACH Sim): A tool for systematic simulation scenario design. Sim Healthcare. 2015; 10: 21-30.

7. Maestre JM, Rudolph JW. Teorías y estilos de debriefing: el método con buen juicio como herramienta de evaluación formativa en salud. Rev Esp Cardiol. 2015; 68 (4): 282-285.

8. Powell C. The Delphi technique: myths and realities. J Adv Nurs. 2003; 41 (4): 376-382.

9. https://harvardmedsim.org. [Último acceso 8 de julio de 2019].

10. Dieckmann P, Lippert A, Glavin R, Rall M. When things do not go as expected: scenario life savers. Sim Healthcare. 2010; 5: 219-225.

11. Bambini D. Writing a simulation scenario: a step-bystep guide. AACN Advanced Critical Care. 2016; 27 (1): 62-70.

12. Lai F, Entin E, Dierks M, Raemer D, Simon R. Designing simulation-based training scenarios for emergency medical first responders. In: Proceedings of the Human Factors and Ergonomics Society 48th Annual Meeting. New Orleans: 2004. pp. 1670-1674.

13. Waxman KT. The development of evidence-based clinical simulation scenarios: guidelines for nurse educators. J Nurs Educ. 2010; 49 (1): 29-35.

14. Munroe B, Thomas B, Curtis K, Morris R. Designing and implementing full immersion simulation as a research tool. Australas Emerg Nurs J. 2016; 19: 90-105.

Declaración de intereses: Los autores no tienen relaciones financieras con ninguna empresa comercial de productos o servicios relacionados con la simulación. El Hospital Virtual Valdecilla está afiliado con el Center for Medical Simulation; ambas son instituciones docentes sin ánimo de lucro que ofrecen programas de formación de instructores de simulación con matrícula a través del Institute for Medical Simulation.
Correspondencia: José M Maestre Hospital Virtual Valdecilla. Av. de Valdecilla s/n, 39008, Santander, España. E-mail: jmmaestre@hvvaldecilla.es 